\title{
Dynamics of taut strings undergoing large changes of tension caused by a force-driven traveling mass
}

\author{
Manuel Ferretti a,b , Giuseppe Piccardo ${ }^{\mathrm{a}, \mathrm{c}}$, Francesco dell'Isola ${ }^{\mathrm{a}, \mathrm{b}}$, \\ Angelo Luongo $\mathrm{a}^{\mathrm{a}, \mathrm{b}, *}$ \\ a International Research Center on Mathematics and Mechanics of Complex Systems, University of L'Aquila, 67100 L'Aquila, Italy \\ ${ }^{\mathrm{b}}$ Department of Civil, Construction-Architectural and Environmental Engineering, University of L'Aquila, 67100 L'Aquila, Italy \\ c Department of Civil, Chemical and Environmental Engineering, University of Genoa, 16145 Genova, Italy
}

\begin{abstract}
A B S T R A C T
The nonlinear dynamics of a horizontal, initially taut, elastic string, subjected to a trav-eling point-mass driven by an assigned time-dependent force, is studied. A kinematically exact nonlinear model for the free boundary problem, previously derived by the authors through a variational procedure, is resumed. The model is developed in the framework of a mixed formulations, ruling the time-evolution of the configuration variables and the reactive contact forces. The model is then consistently simplified, via a static condensa-tion of the horizontal displacements of the string. Differently from most of papers in lit-erature, here the dynamic tension is accounted in the equations of motion, as a nonlin-ear effect. The simplified equations are discretized by the Galerkin method, which leads to a set of ordinary differential equations in the amplitudes of the trial functions and in the unknown abscissa of the pointmass. As a further simplified model, the mass of the string is neglected, and a set of two ordinary differential equations in the coordinates of the point-mass is derived. Numerical results are obtained, aimed at exploring the influ-ence of the mass of the string and of the dynamic tension. The focus is on analyzing pecu-liar 'back and forth' motions of the pointmass, when this is driven by small or even zero forces.
\end{abstract}

\section{Introduction}

The problem of a point-mass traveling a taut string is a classical topic which attracted many scientists since a long time ago [1,2]. Most of these studies, with rare exceptions, concern the case in which the point-mass travels the string with assigned uniform velocity, the problem being formulated and solved in a purely linear context, see e.g. Refs. [1,3]. The classical assumption consists in supposing that the pretension of the string remains unchanged during the motion, i.e. the dynamic tension is considered negligible. By using this model, Smith, in a fundamental paper (1964), commented an amazing paradox, said of Smith-Stokes [1,4], namely that the point-mass reaches the right end of the string at a height different from that of support. Solutions exhibit a singular behavior as the mass approaches the right-hand support (e.g., jump of the mass displacement [3]), leading to reactive forces and per-unit-length energy unbounded near the support. Differently from previous works, Smith's

\footnotetext{
* Corresponding author. International Research Center on Mathematics and Mechanics of Complex Systems, University of L'Aquila, 67100 L'Aquila, Italy. E-mail addresses: manuel.ferretti@univaq.it (M. Ferretti), giuseppe.piccardo@unige.it (G. Piccardo), francesco.dellisola@uniroma1.it (F. dell'Isola), angelo.luongo@univaq.it (A. Luongo).
} 
paper points out that this result arises even in the presence of inertia, at least for the case of string. Nowadays this paradox has not been explained, yet.

Some significant papers on the subject, drawn from the literature, are recalled here. In Refs. [5,6], transition radiations of elastic waves are analyzed and discussed in detail; they are generated by a mechanical object experiencing a uniform rectilinear motion along an inhomogeneous elastic system. Metrikine [7] proposed a method to find dynamic instability zones of a moving particle on a periodically supported, infinitely long elastic string. In Ref. [3], the paradoxical properties of the solution of a string under a traveling single moving mass are analyzed. A formulation that reduces the problem to a second-order matrix ordinary differential equation is proposed, and interesting numerical results about the paradox are discussed. In Refs. [8,9], the same authors propose an efficient space-time finite element method approach for the numerical analysis of the problem. Finally, in Ref. [10] particular attention is devoted to the singularity that the mass induce in the slope of the string. In that paper, in order to better describe the singularity, the time-dependent domain has been transformed into a time-invariant domain, via a suitable coordinate transformation, and the continuous problem has been discretized via the Galerkin method by using a proper set of trial functions.

Nonlinear systems, in contrast, received little attention; some examples are the following. In Ref. [11], cables with moving mass are investigated. In particular, by introducing quasi-static assumptions, a nonlinear condensed planar model, accounting for the effect of the moving mass, is derived and, after linearization, its transient linear planar dynamics is studied. In Ref. [12], the dynamics of an elastic cable subjected to a traveling moving mass particle is investigated. The equations of motions are obtained via the Hamilton principle and then discretized via a Galerkin approach; several examples are developed and the results obtained by Fourier and wavelet representations are compared, showing that wavelets are able to minimize the spurious oscillations.

A kinematically exact nonlinear model was formulated in Ref. [13], in which the horizontal motion of the point-mass is firstly considered unknown, while a horizontal driving force is assigned. Consequently, a new equation ruling the evolution of the abscissa occupied by the mass is established. This 'extended' model was successively simplified, under the assumption that the dynamic tension is negligible, and the mass of the string ignored. The model highlights the existence of a horizontal reactive force at the mass-string contact, which assumes the meaning of a 'wave resistance force' that, under certain circumstances, prevents the mass to reach the right support, causing, in contrast, peculiar 'back and forth' motions.

A new 'minimal' model was developed in Ref. [14]. By still retaining negligible the dynamic tension, here the mass of the string was accounted for in the simplified nonlinear model, and the problem recast in a form of an integrodifferential system. However, the dynamic tension was post-evaluated as a higher-order effect, based on the lower-order response, according to a perturbation procedure. This approach permitted to check that 'back and forth' motions exist for small driving force and large vertical load. But, in contrast with the hypothesis, this behavior causes large dynamic tensions, such as to raise doubts about the actual existence of such kind of motions. Further studies must therefore be carried out in order to include the dynamic tension in the equations of motion. As another important task, in the case of assigned horizontal motion of the mass, large tensions and displacements were found in the boundary layer close to the right-end support when the mass approaches it, thus revealing the importance of the dynamic component of the string tension.

The present paper is an attempt to give an answer to the open questions, still using a simplified model, but 'improved' with respect to the minimal model. Namely, the dynamic tension is accounted for in the equations of motion. A Galerkin solution is pursued for the massive model, and a semi-analytical solution is implemented for the massless model. Both cases of assigned horizontal motion and driving force are considered. Attention is devoted to the Stokes/Smith paradox [1,4] and 'back and forth' motions.

\section{Exact massive model}

Let us consider the dynamics of a horizontal, massive and weightless taut string, under a traveling point-mass guided by a horizontal driving force $D$ of known and time-depending intensity (Fig. 1). The relevant equations of motion can be derived by the Hamilton principle [14]. The Hamilton functional, accounting for kinematic constraints, reads:

$$
\begin{aligned}
\boldsymbol{H}\left[x, y, u, v, \xi, R_{x}, R_{y}\right]:= & \int_{t_{1}}^{t_{2}}\left[\frac{1}{2} M\left(\dot{x}^{2}+\dot{y}^{2}\right)+\frac{1}{2} \int_{0}^{\ell} m\left(\dot{u}^{2}+\dot{v}^{2}\right) \mathrm{d} s-\int_{0}^{\ell} \phi(\varepsilon) \mathrm{d} s\right] \mathrm{d} t \\
& +\int_{t_{1}}^{t_{2}}\left[D x-P y-R_{x}(x-\xi-u(\xi, t))-R_{y}(y-v(\xi, t))\right] \mathrm{d} t
\end{aligned}
$$

where $M$ is the point-mass, with $x(t), y(t)$ its coordinate at the time $t$; dots denote time-differentiation; $m$ is the mass per unit length of the string; $u(s, t), v(s, t)$, with $s \in[0, \ell]$ a material abscissa, are the horizontal and vertical displacement components of the string, respectively, measured from the prestressed configuration;

$$
\phi(\varepsilon):=T_{0} \varepsilon+\frac{1}{2} E A \varepsilon^{2}
$$




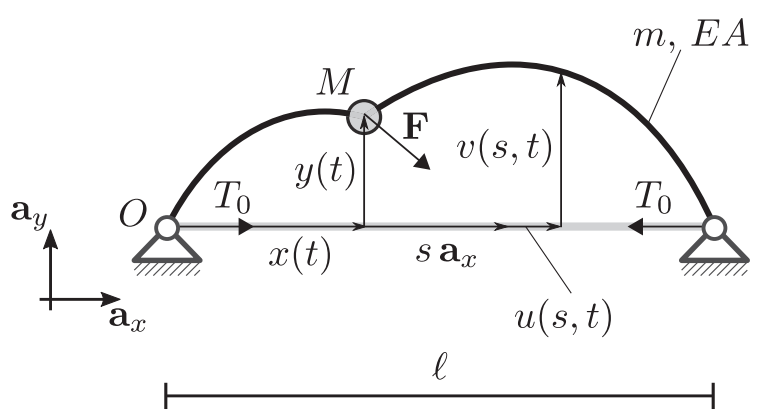

Fig. 1. Horizontal taut string under a traveling heavy point-mass; $\mathbf{F}=D(t) \mathbf{a}_{x}-P(t) \mathbf{a}_{y}$, $D$ being the driving force and $P$ a downward dead load.

is the elastic energy of the string, in which $T_{0}$ is the pretension and $E A$ is the axial stiffness, with:

$$
\varepsilon=\sqrt{\left(1+u^{\prime}(s, t)\right)^{2}+v^{\prime 2}(s, t)}-1,
$$

the (exact) unit extension, where primes denote space-differentiation; $D(t)$ is the driving force, $P$ a downward dead load, including the weight $M g$ of the point-mass; $R_{x}(t), R_{y}(t)$ are two Lagrangian multipliers, having the meaning of horizontal and vertical reactive forces exchanged between the point-mass and the string, which enforce the compatibility conditions of permanent contact between the two bodies. These latter conditions state that the position of the mass equates that of the string at the material abscissa $\xi(t)$ instantaneously occupied. Since $\xi(t)$ is unknown, the problem has the form of a free boundary problem.

From the Green law, the current tension $T=\frac{\partial \phi}{\partial \varepsilon}$ follows:

$$
T=T_{0}+E A \varepsilon(s, t),
$$

i.e. it is made of a known static component $T_{0}$ and an unknown dynamic component $\widetilde{T}:=E A \varepsilon(s, t)$.

By applying the variational calculus, and accounting for the movable extremes of integration, the following Euler-Lagrange equations are drawn (see Ref. [14]):

$$
\begin{aligned}
& {\left[\frac{T}{1+\varepsilon}\left(1+u^{\prime}\right)\right]^{\prime}=m \ddot{u},} \\
& {\left[\frac{T}{1+\varepsilon} v^{\prime}\right]^{\prime}=m \ddot{v},} \\
& D-R_{x}=M \ddot{x}, \\
& -P-R_{y}=M \ddot{y}, \\
& \llbracket\left(\frac{T}{1+\varepsilon}-m \dot{\xi}^{2}\right)\left(1+u^{\prime}\right) \rrbracket+R_{x}=0, \\
& \llbracket\left(\frac{T}{1+\varepsilon}-m \dot{\xi}^{2}\right) v^{\prime} \rrbracket+R_{y}=0, \\
& \llbracket \varepsilon+\frac{1}{2} \varepsilon^{2} \rrbracket=0, \\
& x=\xi+u(\xi, t), \\
& y=v(\xi, t),
\end{aligned}
$$

in which the Hadamard conditions:

$$
\begin{aligned}
\llbracket \dot{u} \rrbracket+\dot{\xi} \llbracket u^{\prime} \rrbracket & =0, \\
\llbracket \dot{v} \rrbracket+\dot{\xi} \llbracket v^{\prime} \rrbracket & =0,
\end{aligned}
$$

have been accounted for, and where the double square brackets denotes the jump of the argument at the singular point $\xi(t)$ (e.g. $\left.\llbracket v^{\prime} \rrbracket=v^{\prime}\left(\xi_{+}, t\right)-v^{\prime}\left(\xi_{-}, t\right)\right)$. Eq. (5) are the field equations, governing the motion of the string; Eq. (6) are the equations of motion of the point-mass; Eq. (7) are the boundary conditions at the singularity, expressing the balance of forces and momentum 
of an infinitesimal neighborhood of singularity; Eq. (8) is a further boundary conditions at the singularity, expressing balance of energy (see Ref. [13] for details); finally Eq. (9) are compatibility conditions.

These equations must be accompanied by the geometric boundary and continuity conditions:

$$
\begin{aligned}
u(0, t) & =u(\ell, t)=0, \\
v(0, t) & =v(\ell, t)=0, \\
\llbracket u \rrbracket & =0, \\
\llbracket v \rrbracket & =0,
\end{aligned}
$$

together with the initial conditions:

$$
\begin{aligned}
& u(s, 0)=0, \quad \dot{u}(s, 0)=0, \\
& v(s, 0)=0, \quad \dot{v}(s, 0)=0, \\
& x(0)=0, \quad \dot{x}(0)=\dot{\xi}_{0}, \\
& y(0)=0, \quad \dot{y}(0)=0, \\
& \xi(0)=0, \quad \dot{\xi}(0)=\dot{\xi}_{0},
\end{aligned}
$$

in which the string is considered at the rest at $t=0$, when the point-mass is on the left end.

\section{A simplified massive nonlinear model}

A simplified nonlinear model is formulated, based on the following hypotheses: (h1) prevalently vertical displacements, $u \ll v$; (h2) small strain $\varepsilon \ll 1$, entailing $u^{\prime} \ll 1$; (h3) small pretension-to-stiffness ratio, $T_{0} \ll E A$; (h4) large dynamic tension $\widetilde{T} \geq O\left(T_{0}\right)$. This model will be named 'improved', with respect to the 'minimal' model formulated in a companion paper [14], in which the dynamic tension is neglected. Comparisons of results supplied by the two models will be given ahead.

\subsection{The horizontal motion of the string and the dynamic tension}

The hypothesis (h3) allows neglecting the horizontal inertia force - mü in Eq (5-a), since the longitudinal wave celerity is much higher than the transverse wave celerity. By using (h2), it follows from the same equation, that $T$ is step-wise constant in the domain. However, (h2) also entails Eq. (8) simplifies into $\llbracket \varepsilon \rrbracket=0$, so that $T$ is strictly constant in space, i.e. $\widetilde{T}=\widetilde{T}(t)$.

The series expansion of the strain (3) reads:

$$
\varepsilon=u^{\prime}+\frac{1}{2} v^{\prime 2} \text {. }
$$

Integration of the previous equation with $\varepsilon=$ const, the boundary conditions $u(0)=u(\ell)=0$ and $\llbracket \varepsilon \rrbracket=0$, leads to:

$$
u=-\frac{1}{2} \int_{0}^{s} v^{\prime 2}(\check{s}, t) \mathrm{d} \check{s}+\frac{s}{2 \ell} \int_{0}^{\ell} v^{\prime 2}(s, t) \mathrm{d} s,
$$

expressing that the horizontal displacement is a passive variable, 'slave' of the vertical displacement.

Consequently, the dynamic tension reads:

$$
\widetilde{T}=\frac{E A}{2 \ell} \int_{0}^{\ell} v^{\prime 2}(s, t) \mathrm{d} s .
$$

\subsection{The vertical motion}

The vertical motion is governed by the second of Eqs. (5)-(7) and (9). With the previous hypotheses and results, and by retaining only the leading terms in each equations, Eqs. (5) and (7) become:

$$
\begin{aligned}
& \left(T_{0}+\widetilde{T}\right) v^{\prime \prime}=m \ddot{v}, \\
& \left(T_{0}+\widetilde{T}-m \dot{\xi}^{2}\right) \llbracket v^{\prime} \rrbracket+R_{y}=0 .
\end{aligned}
$$

The previous equations can be merged, as customary, in a single equation, in which the Dirac delta appears. By appending to it the remaining Eqs. (6) and (9), it follows: 


$$
\begin{aligned}
& \left(T_{0}+\widetilde{T}\right) v^{\prime \prime}+R_{y} \delta(s-\xi)=m \ddot{v}, \\
& -P-R_{y}=M \ddot{y}, \\
& y=v(\xi, t) .
\end{aligned}
$$

These equations govern the vertical motion of the string and mass according to a mixed formulation, in which a reactive force appears together with displacements. If they are combined to eliminate $R_{y}$ and $y$, the following single equation, pure in $v(s, t)$ and $\xi(t)$, is obtained:

$$
\left(T_{0}+\widetilde{T}\right) v^{\prime \prime}=\left[P+M\left(\ddot{\xi} v_{ \pm}^{\prime}+\dot{\xi}^{2} v_{ \pm}^{\prime \prime}+2 \dot{\xi} \dot{v}_{ \pm}^{\prime}+\ddot{v}_{ \pm}\right)\right] \delta(s-\xi)+m \ddot{v},
$$

in which the subscript \pm denotes evaluation either on the left or on the right of the singularity. Eq. (21) generalizes a well-known result of literature (see e.g. Ref. [13]), by accounting for changes in tension. It should noticed that, if the horizontal motion $x$ of the point-mass is assigned, since $x \simeq \xi$, it is known $\xi=\bar{\xi}(t)$; then, Eq. (21) (with relevant boundary and initial conditions) determines the string response by itself.

\subsection{The horizontal motion of the mass}

The horizontal motion of the mass is governed by the first of Eqs. (6), (7) and (9). In the framework of the approximation adopted, Eq. (7) reads:

$$
\left(T_{0}+\widetilde{T}-m \dot{\xi}^{2}\right) \llbracket u^{\prime} \rrbracket+R_{x}=0 .
$$

Since $\llbracket \varepsilon \rrbracket=0$, from Eq. (13) it follows:

$$
\llbracket u^{\prime} \rrbracket=-\frac{1}{2} \llbracket v^{\prime 2} \rrbracket \equiv-\llbracket v^{\prime} \rrbracket\left\langle v^{\prime}\right\rangle,
$$

where $\left\langle v^{\prime}\right\rangle:=\frac{1}{2}\left(v_{+}^{\prime}+v_{-}^{\prime}\right)$; therefore, from Eqs. (22) and (17):

$$
R_{x}=-R_{y}\left\langle v^{\prime}\right\rangle \text {. }
$$

Thanks to this result, and neglecting $\ddot{u}$, Eqs. (6) and (9) can be combined into:

$$
D+R_{y}\left\langle v^{\prime}\right\rangle=M \ddot{\xi} .
$$

This equation (coupled with Eqs. (18)-(20)) rules the horizontal motion of the mass in terms of mixed variables. Alternatively, the same equation can be written in terms of kinematic variables only, with the help of Eqs. (19) and (20), i.e.:

$$
D-\left[P+M\left(\ddot{\xi} v_{ \pm}^{\prime}+\dot{\xi}^{2} v_{ \pm}^{\prime \prime}+2 \dot{\xi} \dot{v}_{ \pm}^{\prime}+\ddot{v}_{ \pm}\right)\right]\left\langle v^{\prime}\right\rangle=M \ddot{\xi},
$$

which supplements Eq. (21). If the horizontal motion of the mass is assigned, this equation allows determining the driving force $D=D(t)$, after the vertical response $v(s, t)$ has been evaluated.

It is important to remark that Eq. (24) for the wave pressure force was obtained for the first time by Nicolai [15] and, after many years, rediscovered in studies by Vesnitskiy et al. (see e.g., Ref. [16]). Then, its nature became the subject of subsequent recent discussions, see e.g. Refs. [17-19].

\subsection{Final nondimensional governing equations}

By summarizing previous results, the nonlinear model in terms of displacements is constituted by Eqs. (15), (21) and (26), for the unknowns $v(s, t), \xi(t), \widetilde{T}(t)$. By introducing the nondimensional quantities:

$$
\begin{aligned}
& \hat{s}:=\frac{s}{\ell}, \quad \hat{t}:=\frac{1}{\ell} \sqrt{\frac{T_{0}}{m}} t, \quad \hat{v}:=\frac{v}{\ell}, \quad \hat{\xi}:=\frac{\xi}{\ell}, \quad \mu:=\frac{M}{m \ell}, \\
& \widehat{D}:=\frac{D}{T_{0}}, \quad \widehat{P}:=\frac{P}{T_{0}}, \quad \widehat{R}_{y}:=\frac{R_{y}}{T_{0}}, \quad \hat{\widetilde{T}}:=\frac{\widetilde{T}}{T_{0}}, \quad \alpha:=\frac{E A}{2 T_{0}},
\end{aligned}
$$

the equations read: 


$$
\begin{aligned}
& (1+\widetilde{T}) v^{\prime \prime}=\left[P+\mu\left(\ddot{\xi} v_{ \pm}^{\prime}+\dot{\xi}^{2} v_{ \pm}^{\prime \prime}+2 \dot{\xi} \dot{v}_{ \pm}^{\prime}+\ddot{v}_{ \pm}\right)\right] \delta(s-\xi)+\ddot{v} \\
& D-\left[P+\mu\left(\ddot{\xi} v_{ \pm}^{\prime}+\dot{\xi}^{2} v_{ \pm}^{\prime \prime}+2 \dot{\xi} \dot{v}_{ \pm}^{\prime}+\ddot{v}_{ \pm}\right)\right]\left\langle v^{\prime}\right\rangle=\mu \ddot{\xi} \\
& \widetilde{T}=\alpha \int_{0}^{1} v^{\prime 2}(s, t) \mathrm{d} s
\end{aligned}
$$

in which hat has been omitted, and primes and dots denote differentiation with respect to the nondimensional independent variables. In the same variables, the relevant boundary and initial conditions are:

$$
\begin{aligned}
& v(0, t)=0, \quad v(1, t)=0, \\
& v(s, 0)=0, \quad \dot{v}(s, 0)=0, \\
& \xi(0)=0, \quad \dot{\xi}(0)=\dot{\xi}_{0} .
\end{aligned}
$$

After the problem has been solved, the nondimensional horizontal displacement and the horizontal reaction are post-evaluated as:

$$
\begin{aligned}
& u=-\frac{1}{2} \int_{0}^{s} v^{\prime 2}(\check{s}, t) \mathrm{d} \check{s}+\frac{s}{2} \int_{0}^{1} v^{\prime 2}(s, t) \mathrm{d} s, \\
& R_{x}=\left[P+\mu\left(\ddot{\xi} v_{ \pm}^{\prime}+\dot{\xi}^{2} v_{ \pm}^{\prime \prime}+2 \dot{\xi}_{ \pm}^{\prime}+\ddot{v}_{ \pm}\right)\right]\left\langle v^{\prime}\right\rangle .
\end{aligned}
$$

Then, the motion of the point-mass can be computed as $y=v(\xi, y)$ and $x=\xi+u(\xi, y)$. It is worth noticing that, due the nondimensionalization adopted for time, the nondimensional velocities assume the meaning of dimensional velocities divided by the transverse celerity of the string. In this paper, they will be considered to be less than 1 (i.e. only subsonic motions will be analyzed).

\section{Discretized massive model}

A discretized model for the massive string is obtained by the Galerkin Method. Accordingly, the transverse displacement is expressed through the following truncated series:

$$
v(s, t)=\sum_{j=1}^{N_{e}} \phi_{j}(s) q_{j}(t),
$$

where the trial functions $\phi_{j}(s):=\sqrt{2} \sin \left(\omega_{j} s\right)$, with $\omega_{j}:=j \pi, j=1,2,3, \cdots$ are the eigenfunctions of the linear taut string, suitably normalized. Since the system consists of a partial differential Eq. (28), an ordinary differential equation in the $\xi$ variable (29) and an integral Eq. (30), the procedure calls for substituting the expansion in the governing set, and weighting the residual in the partial differential equation only, by using the same eigenfunction $\phi_{i}$. The following equations are obtained:

$$
\begin{aligned}
& (1+\widetilde{T}) q_{j} \sum_{j=1}^{N_{e}} \int_{0}^{1} \phi_{i} \phi_{j}^{\prime \prime} \mathrm{d} s=\ddot{q}_{j} \sum_{j=1}^{N_{e}} \int_{0}^{1} \phi_{i} \phi_{j} \mathrm{~d} s \\
& +\left\{\phi_{i}\left[P+\mu \sum_{j=1}^{N_{e}}\left(\ddot{\xi} \phi_{j}^{\prime} q_{j}+\dot{\xi}^{2} \phi_{j}^{\prime \prime} q_{j}+2 \dot{\xi} \phi_{j}^{\prime} \dot{q}_{j}+\phi_{j} \ddot{q}_{j}\right)\right]\right\}_{s=\xi}, \quad i=1, \ldots, N_{e} \\
& D-\left\{\left[P+\mu \sum_{j=1}^{N_{e}}\left(\ddot{\xi} \phi_{j}^{\prime} q_{j}+\dot{\xi}^{2} \phi_{j}^{\prime \prime} q_{j}+2 \dot{\xi} \phi_{j}^{\prime} \dot{q}_{j}+\phi_{j} \ddot{q}_{j}\right)\right] \sum_{j=1}^{N_{e}} \phi_{j}^{\prime} q_{j}\right\}_{s=\xi}=\mu \ddot{\xi}, \\
& \widetilde{T}=\alpha \sum_{h=1}^{N_{e}} \sum_{k=1}^{N_{e}} q_{h} q_{k} \int_{0}^{1} \phi_{h}^{\prime} \phi_{k}^{\prime} \mathrm{d} s .
\end{aligned}
$$

To write Eq. (38), the term $\left\langle v^{\prime}\right\rangle$ has been evaluated. Since the vertical response of the string (36) is smooth, no jumps in $v^{\prime}(s, t)$ can be described. However, as it is well-known in the Fourier theory, for large $N_{e}$ the series converges to the average value of the discontinuity, namely $v^{\prime}(\xi, t) \rightarrow\left\langle v^{\prime}\right\rangle$. 
Using the orthonormalization properties of the trial functions, Eqs. (37)-(39) simplify into:

$$
\begin{aligned}
& \ddot{q}_{i}+\omega_{i}^{2}(1+\widetilde{T}) q_{i}+a_{i} P+\mu\left(\ddot{\xi} \sum_{j=1}^{N_{e}} b_{i j} q_{j}+\dot{\xi}^{2} \sum_{j=1}^{N_{e}} c_{i j} q_{j}+2 \dot{\xi} \sum_{j=1}^{N_{e}} b_{i j} \dot{q}_{j}+\sum_{j=1}^{N_{e}} a_{i j} \ddot{q}_{j}\right)=0, \quad i=1, \ldots, N_{e}, \\
& \mu \ddot{\xi}=D-P-\mu\left(\ddot{\xi} \sum_{j=1}^{N_{e}} b_{j} q_{j}+\dot{\xi}^{2} \sum_{j=1}^{N_{e}} c_{j} q_{j}+2 \dot{\xi} \sum_{j=1}^{N_{e}} b_{j} \dot{q}_{j}+\sum_{j=1}^{N_{e}} a_{j} \ddot{q}_{j}\right) \sum_{k=1}^{N_{e}} b_{k} q_{k}, \\
& \widetilde{T}=\alpha \sum_{h=1}^{N_{e}} \omega_{h}^{2} q_{h}^{2},
\end{aligned}
$$

where:

$$
\begin{array}{lll}
a_{i}:=\left[\phi_{i}\right]_{s=\xi}, & b_{i}:=\left[\phi_{j}^{\prime}\right]_{s=\xi}, & c_{i}:=\left[\phi_{j}^{\prime \prime}\right]_{s=\xi}, \\
a_{i j}:=\left[\phi_{i} \phi_{j}\right]_{s=\xi}, & b_{i j}:=\left[\phi_{i} \phi_{j}^{\prime}\right]_{s=\xi}, & c_{i j}:=\left[\phi_{i} \phi_{j}^{\prime \prime}\right]_{s=\xi} .
\end{array}
$$

Eqs. (40)-(42) represent the discretized model. By substituting $\widetilde{T}$ from Eq. (42) into Eq. (40), the problem is governed by a set of $N_{e}+1$ ordinary differential equations in the unknown $q_{j}, j=1,2, \ldots, N_{e}$ and $\xi$. In the case of assigned horizontal motion, Eq. (40) are decoupled from Eq. (41), that can be utilized for the determination of the unknown $D$.

It is worth noticing that the result obtained by the Galerkin approach cannot be obtained by the Ritz approach. Indeed, the direct substitution of smooth trial function in Eq. (1), and the use of the same simplifying assumptions, lead to an equation of motion in $\xi$ which is decoupled from those of the string. This is due to the fact that the effect of the reactive force at the singular point is lost when the Ritz approach with smooth trial functions is applied.

\section{Massless string model}

A strongly simplified model, which has been often used in the linear context, is the massless string (see, e.g., Refs. [1,13]). Here, it is derived from the improved massive model, in the nonlinear context.

Since a piecewise linear function satisfies the field equation, there is no need to resort to a Galerkin procedure but, in contrast, a semi-analytical solution can be pursued. First, reference is made to the equation of motion cast in mixed form, i.e. Eqs. (18)-(20) and Eq. (25), which involve kinematic variables as well the vertical reaction $R_{y}$, instead of those expressed in terms of the former only. Second, the same nondimensional parameters introduced for the massive model are used, with $m$ the true mass per unit length of string, although the associated inertia forces are neglected. This is done in order to maintain the same nondimensional time for massive and massless models and, therefore, to preserve the meaning of the nondimensional velocity, as a fraction of the celerity of the underlying physical string.

The above recalled equations, in nondimensional form, read:

$$
\begin{aligned}
& (1+\widetilde{T}) v^{\prime \prime}+R_{y} \delta(s-\xi)=0, \\
& -P-R_{y}=\mu \ddot{y}, \\
& y=v(\xi, t), \\
& D+R_{y}\left\langle v^{\prime}\right\rangle=\mu \ddot{\xi},
\end{aligned}
$$

where the inertial $\ddot{v}$ term has been neglected, and $\widetilde{T}$ is defined as in Eq. (30).

Eq. (44) admits the solution:

$$
v(s, t)=\left\{\begin{array}{ll}
c s & 0 \leq s \leq \xi \\
c(1-s) \frac{\xi}{1-\xi} & \xi \leq s \leq 1
\end{array},\right.
$$

where $c$ is an arbitrary constant to be determined via the condition:

$$
(1+\widetilde{T}) \llbracket v^{\prime} \rrbracket+R_{y}=0
$$

This latter, with the solution (48), reads:

$$
\frac{1}{1-\xi} c+\alpha \frac{\xi}{(1-\xi)^{2}} c^{3}-R_{y}=0
$$


which is more easily solved for $R_{y}$ as:

$$
R_{y}=\frac{1}{1-\xi} c+\alpha \frac{\xi}{(1-\xi)^{2}} c^{3} .
$$

On the other hand, substitution of $v(s, t)$ in Eq. (46) yields:

$$
c=\frac{y}{\xi} .
$$

From Eq. (48) it is possible to evaluate $\left\langle v^{\prime}\right\rangle$ in exact form, namely:

$$
\left\langle v^{\prime}\right\rangle=\frac{1-2 \xi}{2(1-\xi)} c
$$

Finally by using Eq. (51) for $R_{y}$, (53) for $\left\langle v^{\prime}\right\rangle$ and (52) for $c$, Eqs. (45) and (47) can be expressed in terms of $y$ and $\xi$ only, i.e.:

$$
\begin{aligned}
& \mu \ddot{y}+\frac{y}{(1-\xi) \xi}+\alpha \frac{y^{3}}{(1-\xi)^{2} \xi^{2}}=-P, \\
& \mu \ddot{\xi}+\frac{(2 \xi-1) y^{2}}{2(1-\xi)^{2} \xi^{2}}+\alpha \frac{(2 \xi-1) y^{4}}{2(1-\xi)^{3} \xi^{3}}=D .
\end{aligned}
$$

Eqs. (54) and (55) are two nonlinear coupled ordinary differential equations, governing the motion of the point-mass. They must be integrated with the initial conditions $y(0)=0, \dot{y}(0)=0, \xi(0)=0$ and $\dot{\xi}(0)=\dot{\xi}_{0}$. Once such equations are solved, the vertical reaction and the displacement field of the string are evaluated via Eqs. (48), (51) and (52); finally, the nondimensional horizontal displacement, the horizontal reaction and the dynamic tension are obtained through Eqs. (24), (30) and (34).

Eqs. (54) and (55) and initial conditions highlight the persistence of the Stokes/Smith paradox, also in the context of the unknown horizontal motion of the point-mass. Indeed, they constitute an initial value Cauchy problem; therefore, when, at an unknown final time $t=t_{f}$, the point-mass occupies the string end point, i.e. $\xi\left(t_{f}\right)=1$ (if any), it is generally $y\left(t_{f}\right) \neq 0$, i.e. the point-mass does not reaches the height of the right-hand support of the string.

\section{Numerical results}

Numerical results are discussed for some case studies, relevant to both (a) assigned and (b) unknown horizontal motion of the point-mass. The massive improved model is prevalently used, but also results provided by the minimal model are shown for comparison (in which the dynamic tension is neglected in the equations of motion, but post-evaluated according to a perturbation scheme, see Ref. [14]). Moreover, the massless string model is occasionally employed for further comparisons. In any cases, a metallic string is considered, having mechanical parameter $\alpha=500$; moreover, in using the Galerkin method, a number $N_{e}=50$ of eigenfunctions is adopted, when not otherwise specified.

\subsection{Assigned horizontal motion}

As a first case study, a point-mass traveling the string with uniform motion $\xi=U t$ is considered, and results achieved by the improved and minimal model (coincident with the classical one, in the assigned motion case) compared in Fig. 2. Here, the static vertical force is $P=0.025$, the mass-ratio $\mu=0.1$ and the velocity-ratio $U=0.9$. The figure shows several nondimensional magnitudes $v s$ the spatial abscissa $x$ instantaneously occupied by the point-mass, obtained by eliminating the time in the parametric equations, namely: (a) the trajectory $y=y(x)$ of the point-mass; (b) the vertical motion of the string at midspan; (c) the unknown driving force, needed to sustain the assigned motion; (d) the vertical reaction exchanged between the pointmass and the string; (e) the dynamic tension; ( $\mathrm{f}$ ) the horizontal displacement of the string at midspan. Black curves refer to the improved model, grey curves to the minimal model. The two models give qualitatively similar, but quantitatively different results. Differences have been found to decrease with the magnitude of the vertical reactive force, which is responsible for the magnitude of displacements and, therefore, of the dynamic tension. In particular, the dynamic tension makes the system stiffer, thus reducing the amplitude of displacements.

When the point-mass approaches the right end, all quantities undergo a sudden change in their qualitative behavior. In particular, the driving force, the vertical reaction and the dynamic tension assume very large values in the boundary layer. This phenomenon has a physical ground, although not yet well understood in the literature, namely that waves reflected between the moving point-mass and the right-end support superimpose themselves in a decreasingly small interval. The improved model confirms this results, but strongly reduces the unrealistic values provided by the minimal model. Moreover, it corrects the erroneous result of the minimal model occurring at the very right side of the trajectory (see the zoom in the figure), according to which, due to the significant horizontal displacements of the string, the horizontal projection of the point-mass exceeds the right end of the string. However, it will be seen further that, for different values of parameters, this odd behavior will be predicted by the improved model too. 


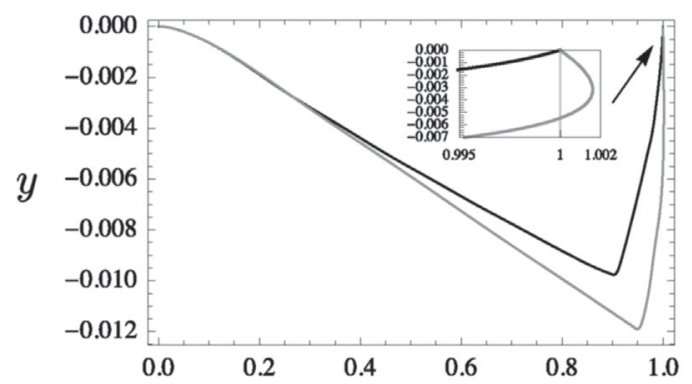

$x$

(a)

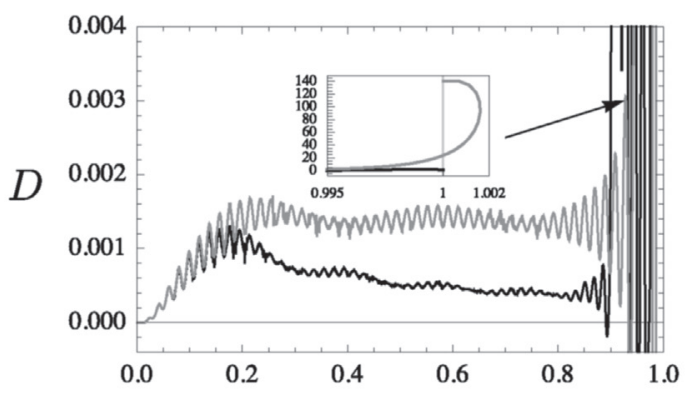

$x$

(c)

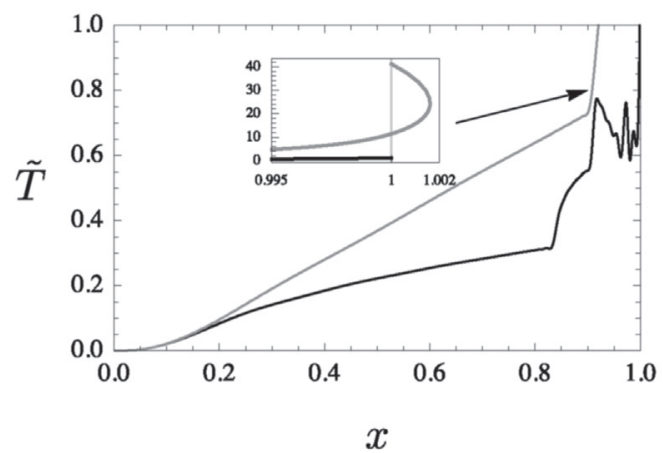

(e)

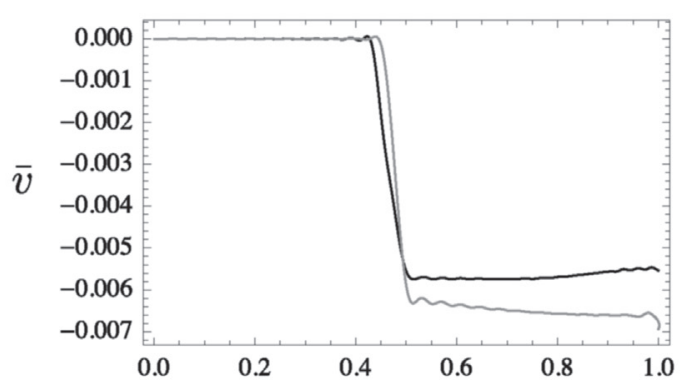

$x$

(b)

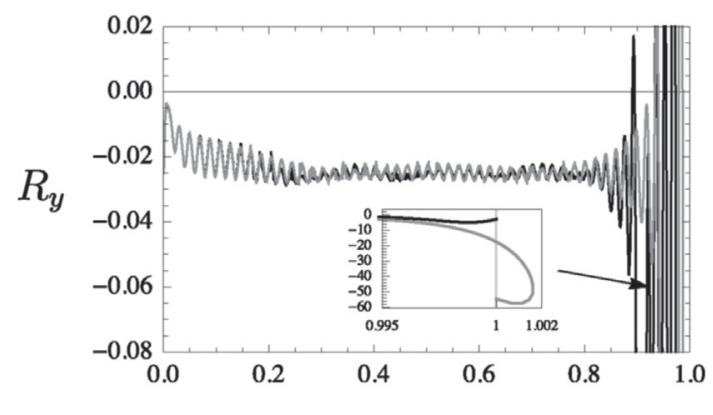

$x$

(d)

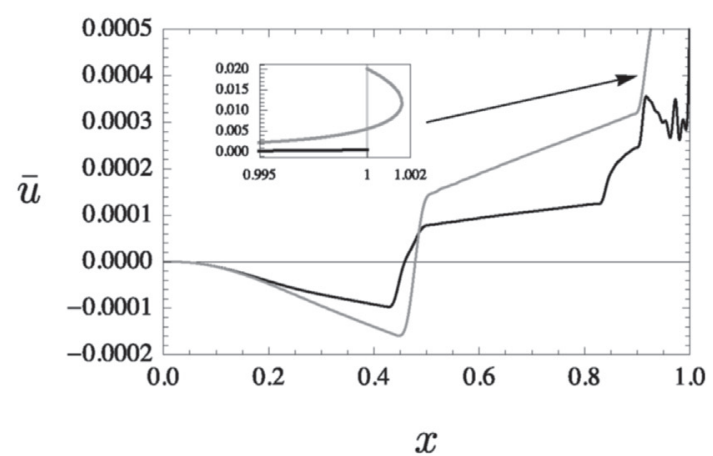

(f)

Fig. 2. Response for the case of assigned horizontal uniform motion, when $P=0.025, \mu=0.1$ and $U=0.9$ : (a) motion of the point mass; (b) $\bar{v}=v\left(\frac{1}{2}, t\right)$; (c) driving force; (d) vertical reactive force; (e) dynamic tension; (f) $\bar{u}=u\left(\frac{1}{2}, t\right)$. In black and grey lines the improved and the minimal model, respectively.

\subsection{Stokes-Smith paradox}

To investigate the Stokes-Smith paradox, the trajectory of the point-mass are carefully observed near the right-end support. A new set of parameters is assumed for the string $(P=0.01, \mu=1)$ and an assigned velocity is considered $(U=0.5)$. A convergence study for increasing number $N_{e}$ of the eigenfunctions is carried out and the results are displayed in Fig. 3 for the minimal and improved massive models. Moreover, the trajectory determined by the massless models is shown for both cases (i.e., dynamic tension neglected or accounted for). This analysis generalizes the one given in Ref. [3] for the classical model. The curves relevant to the massive models show that, since the trial functions all vanish at the right end, the point-mass reaches the target $y=0$. However, when $N_{e}$ is large, this approach occurs via a nearly-vertical tangent for the vertical component of the displacement of the mass, $v(\xi, t)=y$ (see Fig. 3-g,h, showing that the Galerkin solution seems to be an approximation of a trajectory which tends to a value of $y$ different from zero). In addition, the horizontal component of the displacement, $u(\xi, t)$ 


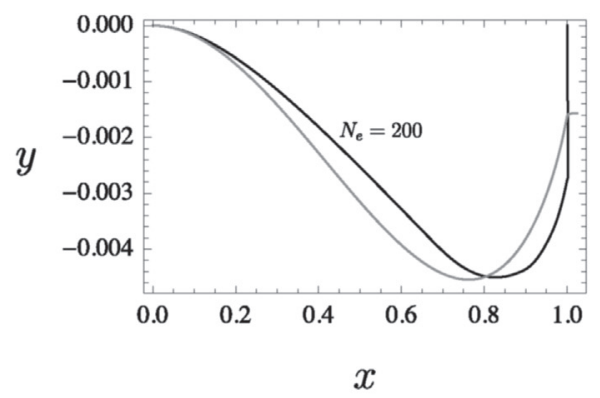

(a)

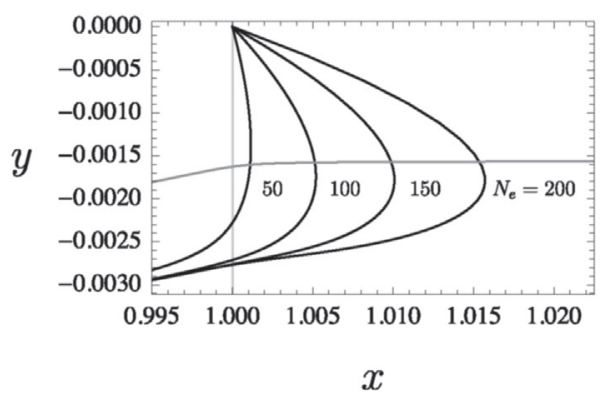

(c)

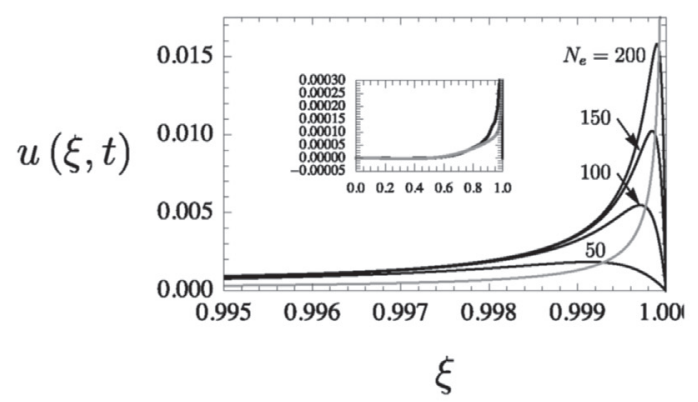

(e)

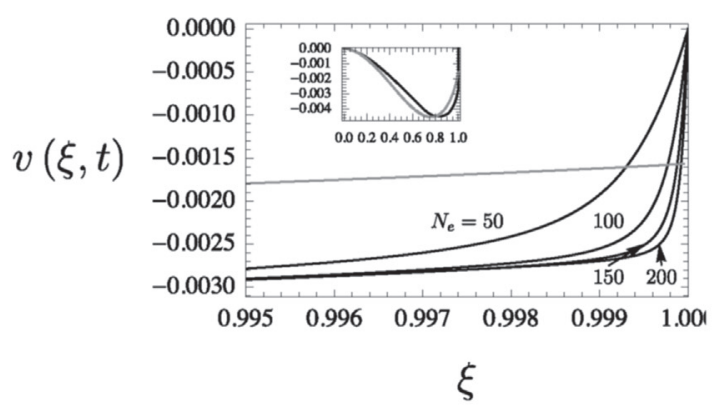

(g)

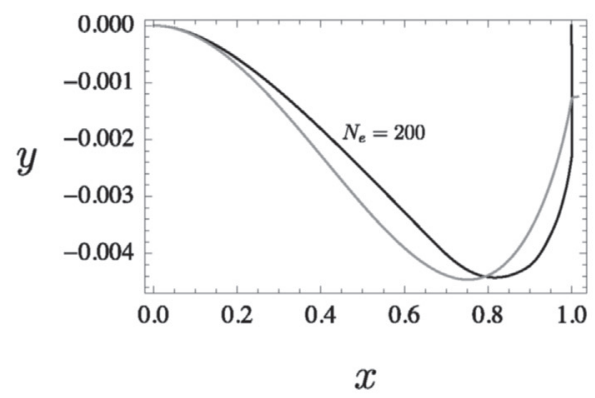

(b)

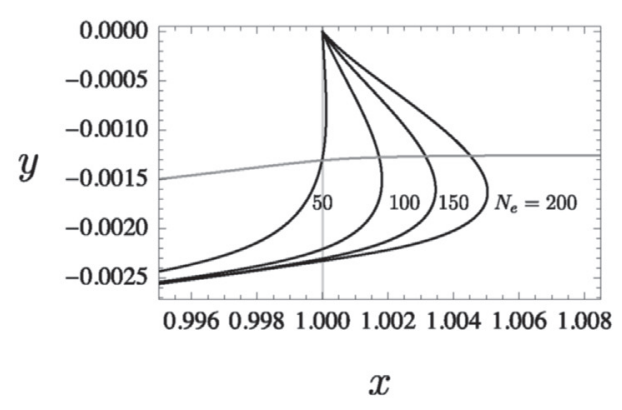

(d)

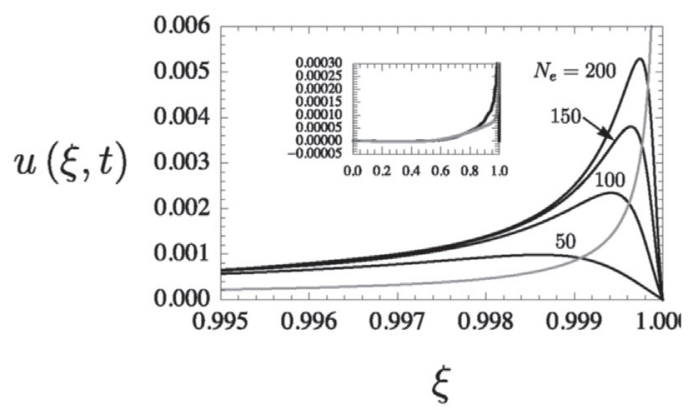

(f)

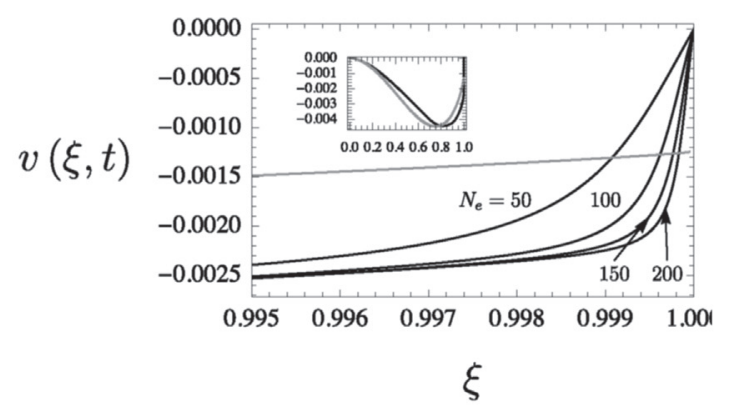

(h)

Fig. 3. Trajectory of the point mass close to the right end for different numbers $N_{e}$ of trial functions, when $P=0.01, \mu=1$ and $U=0.5$ : (a), (c), (e), (g) minimal model, (b), (d), (f), (h) improved model. Black lines refer to the massive model, grey lines to the massless model.

(see Fig. 3-e,f), shows a peak near the right-end support, that becomes larger as the discretization is improved. The behavior pattern is confirmed by the massless model, which is independent of $N_{e}$ (not requiring a Galerkin discretization).

If results supplied by the classical and improved models are compared, it is apparent that the latter reduces the extent of the paradox, but does not eliminate it. 


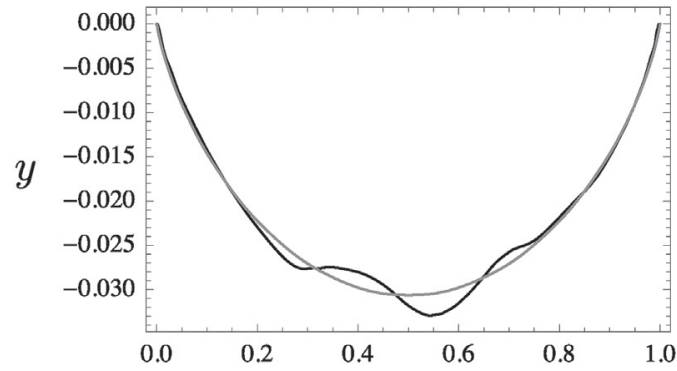

$x$

(a)

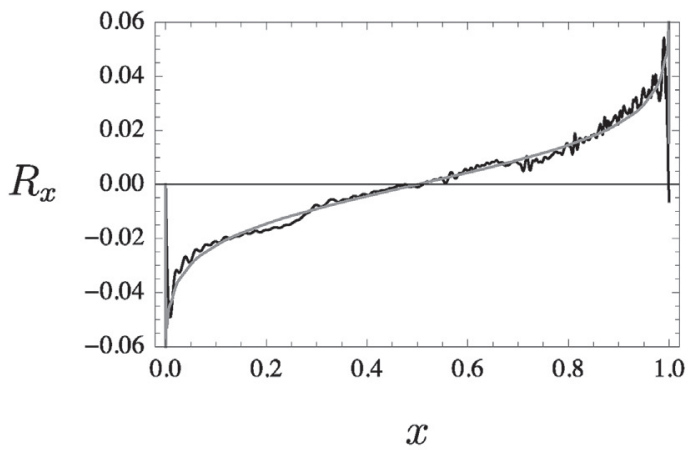

(c)

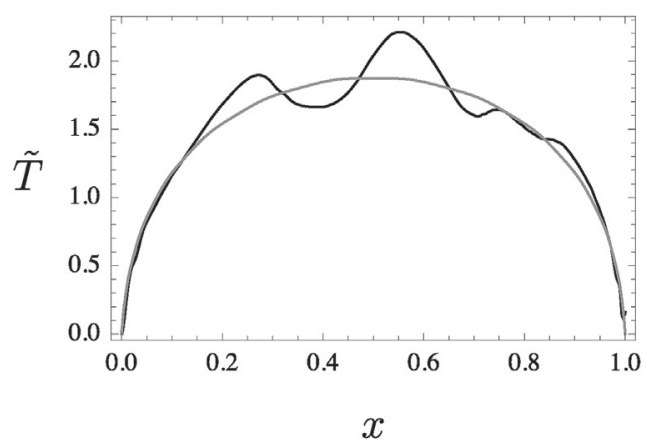

(e)

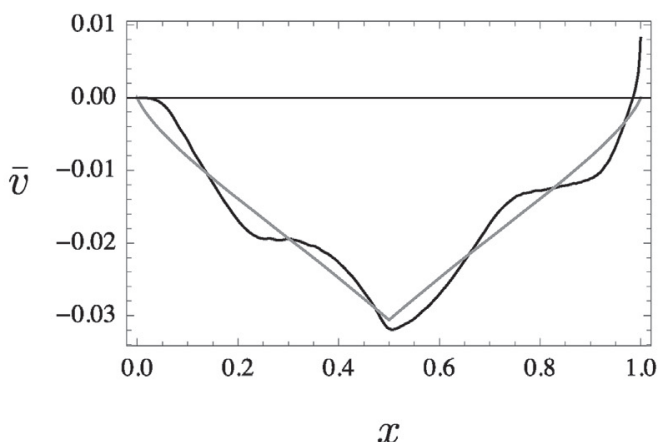

(b)

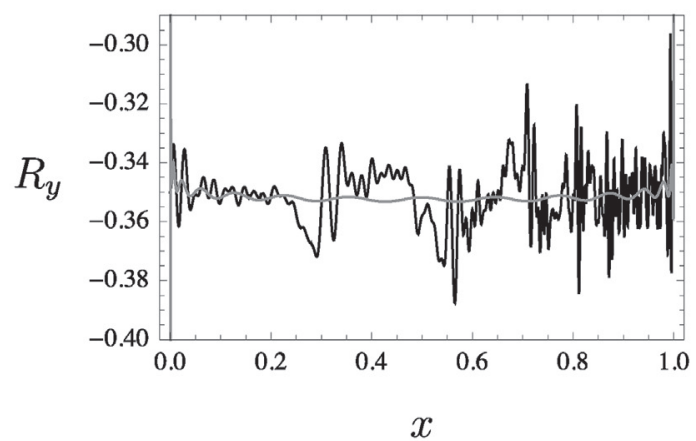

(d)

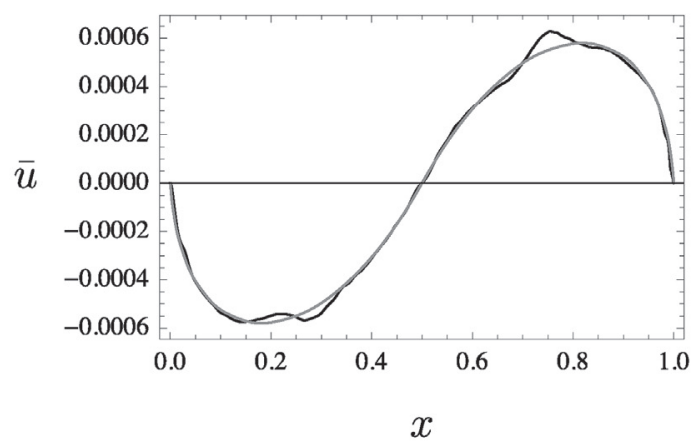

(f)

Fig. 4. Comparison between massive (black lines) and massless (grey lines) taut string models, for the case of assigned driving force, when $\mu=0.1, P=0.35$, $D=2 \times 10^{-4}$ and $\dot{\xi}_{0}=0$ : (a) trajectory of the point mass; (b) $\bar{v}=v\left(\frac{1}{2}, t\right) ;(\mathrm{c})$ horizontal reactive force; (d) vertical reactive force; (d) dynamic tension; (e) $\bar{u}=u\left(\frac{1}{2}, t\right)$.

\subsection{Assigned driving force: monotonic horizontal motions}

A second case-study is devoted to analyze the influence on the response of the distributed mass, when the point-mass is driven by a known and constant horizontal force. The following parameters are chosen: $\mu=0.1, P=0.35, D=2 \times 10^{-4}$ and $\dot{\xi}_{0}=0$, and the response of the massive and massless improved models compared (see Fig. 4). It is seen that the pointmass reaches the right end moving in a monotonic way. The two models supply quite similar results, in terms of displacements and dynamic tension. These behavior patterns suggest that the distributed mass acts as a perturbation of the massless string, inducing small oscillations around the solution of the latter. A similar trend is followed by the horizontal reaction, while the vertical reaction exhibits large oscillations.

The differences between the two models are more evident when one is looking for 'back and forth' horizontal motions of the point mass, which usually occur for large vertical forces $P$, able to trigger sufficiently high horizontal reactions, needed to overcome the driving force and to invert the motion (see the next subsection). It has been found that the massive model experiences oscillatory horizontal motions of the mass at values of $P$ much lower than the massless model. 


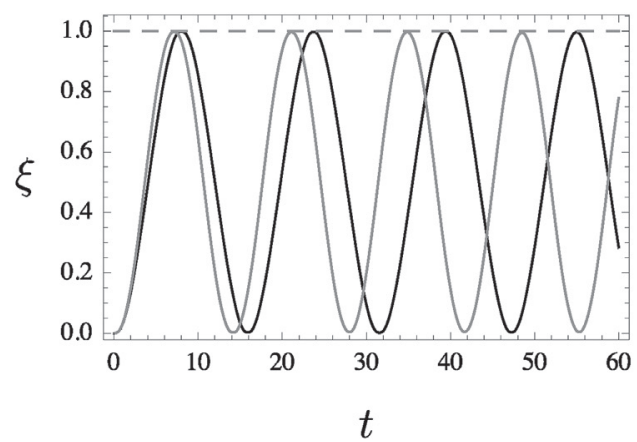

(a)

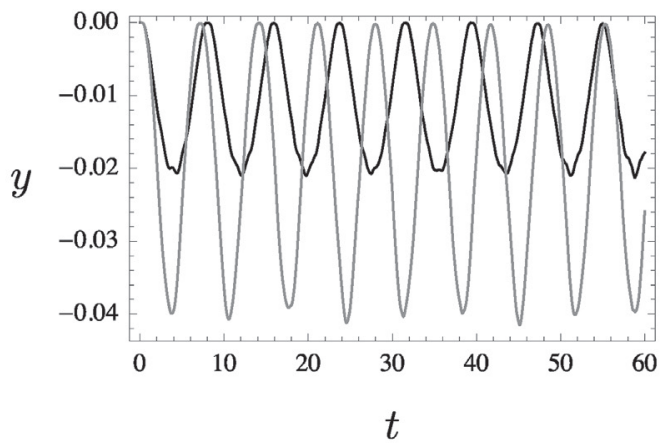

(c)

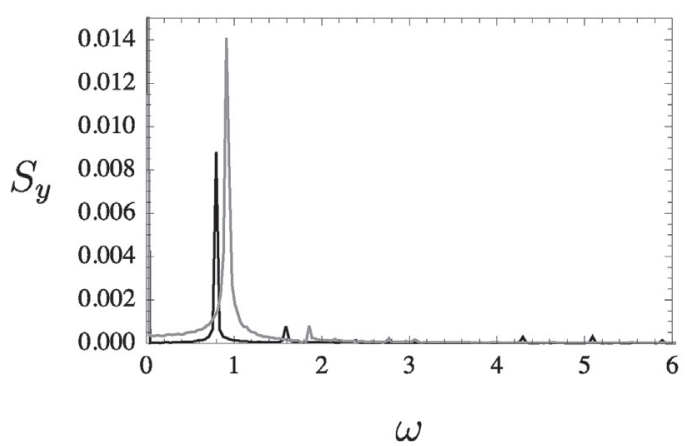

(e)

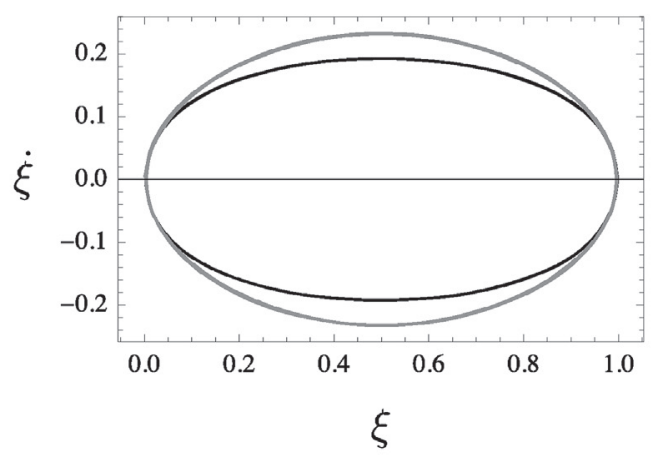

(b)

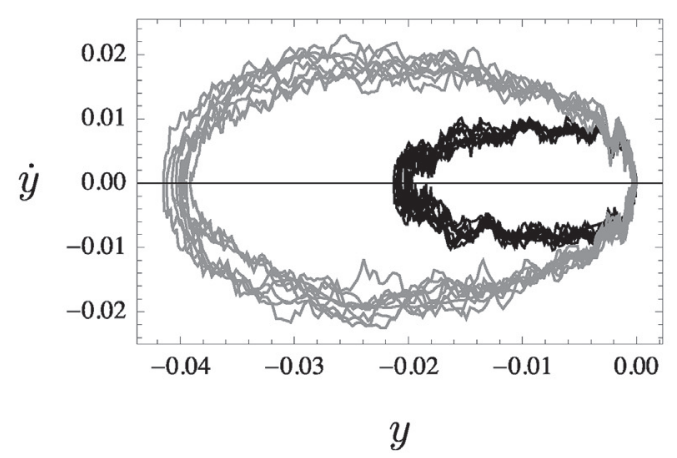

(d)

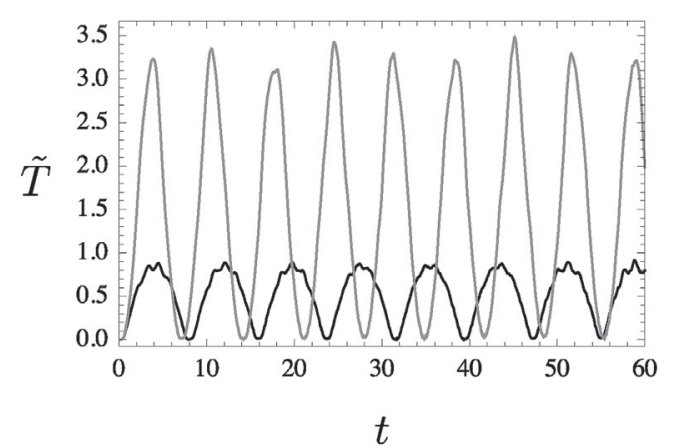

(f)

Fig. 5. Oscillatory motion of the mass between the two supports, when $\mu=0.1, D=0, \dot{\xi}_{0}=0.005$ and $P=0.15$. In black and grey lines the improved and the minimal model, respectively. $S_{y}$ Fourier spectrum of the amplitude of $y(t)$.

\subsection{Assigned driving force: 'back and forth' motions}

A third case-study is devoted to analyze 'back and forth' motions of the point-mass. These motions were discovered for the first time in Ref. [13] and then critically reconsidered in a companion paper [14], where it was found that they are related to high dynamic tensions, neglected at a first stage. To this end, only the massive model is employed, and the improved and minimal models compared.

Two responses have been analyzed, and depicted in Figs. 5 and 6. Both of them concern a mass ratio $\mu=0.1$. In the former problem the point-mass is subjected to a vertical load $P=0.15$, it is free $(D=0)$ but possesses an initial velocity $\dot{\xi}_{0}=0.005$; in the latter problem the mass is vertically loaded by $P=0.7$, driven by a force $D=2 \times 10^{-4}$, and starts from the rest, $\dot{\xi}_{0}=0$. The values of the vertical forces have been selected as the (approximately) smallest values which entail oscillatory motions in the improved model (i.e., smaller values trigger monotonic motions). It is worth noting that, as expected, a larger load $P$ is needed when the point-mass is driven by a horizontal force, in order that the horizontal reaction is able to invert the motion. 


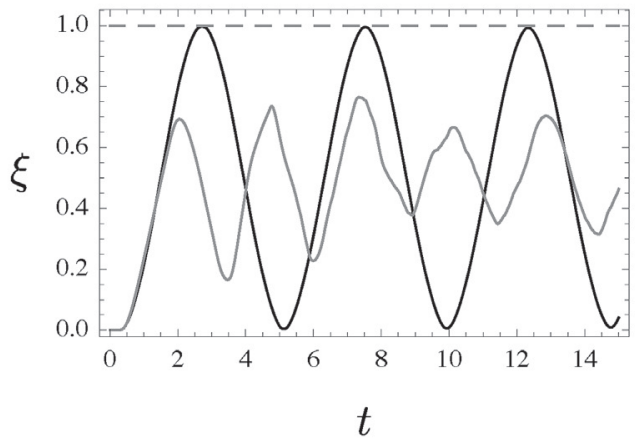

(a)

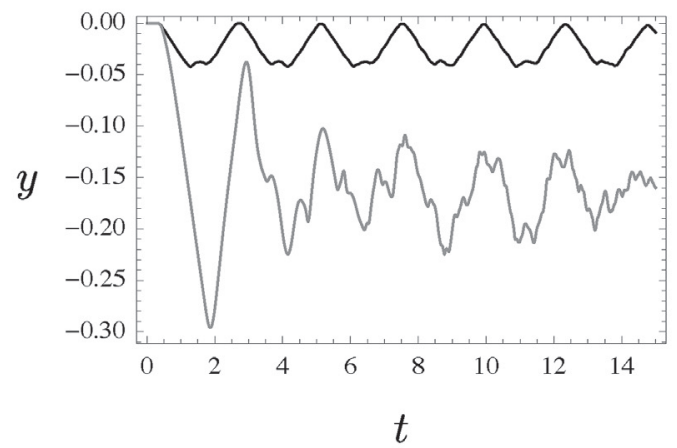

(c)

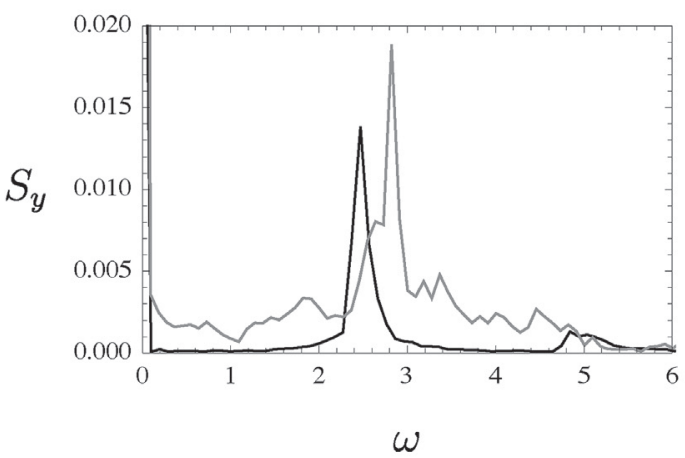

(e)

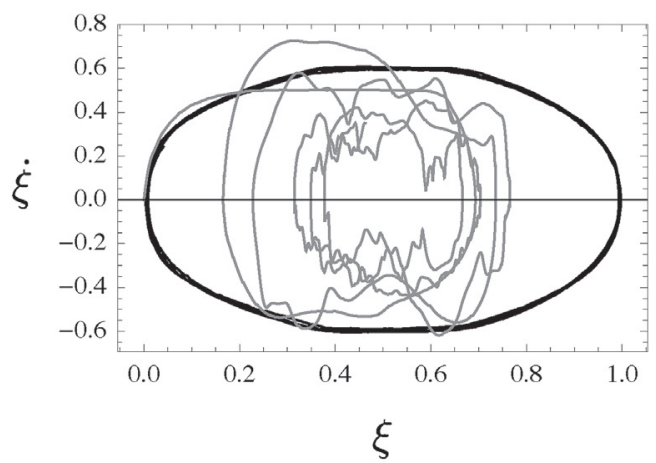

(b)

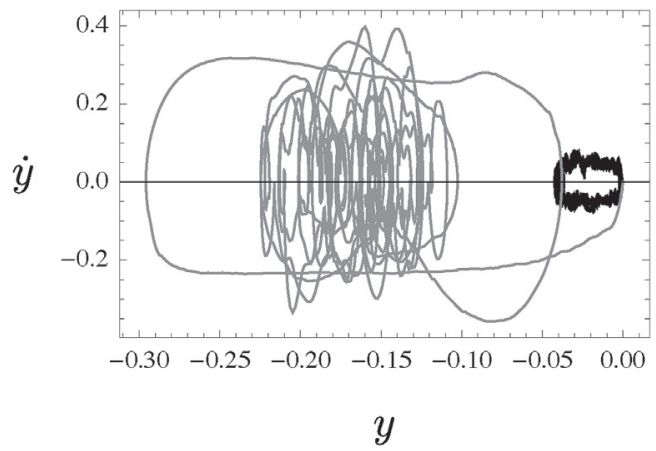

(d)

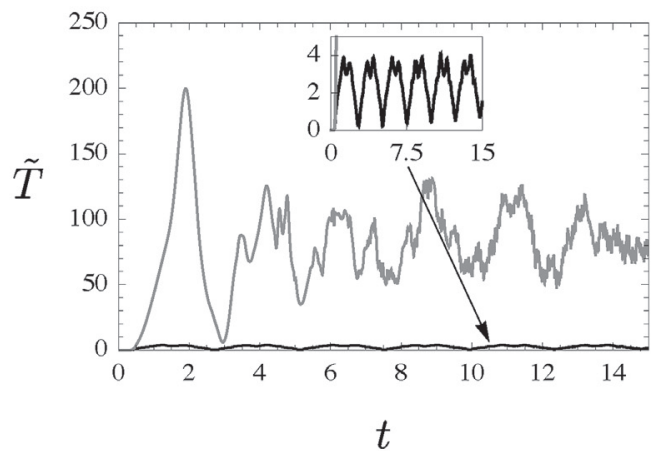

(f)

Fig. 6. Oscillatory motion of the mass between the two supports, when $\mu=0.1, D=2 \times 10^{-4}, \dot{\xi}_{0}=0$ and $P=0.7$. In black and grey lines the improved and the minimal model, respectively. $S_{y}$ Fourier spectrum of the amplitude of $y(t)$.

The figures show the time-histories of the material abscissa $\xi$ occupied by the point-mass and its vertical position $y$, as well that of the dynamic tension $\widetilde{T}$; moreover, the two phase-planes and the Fourier spectrum of the vertical displacement $y$ are also shown. These figures point out how the presence of a sufficiently high vertical load $P$ leads to an evolution of the concept of paradox: the problem is no longer singular in the boundary layer, the point-mass moves 'back and forth' and it is no longer able to approach the right-hand support, continuing to oscillate in an ideal situation of absence of dissipation. The improved model describes almost-periodic motions, whose trajectories are very close to cycles; moreover, the vertical displacements and the dynamic tension have small amplitude. The minimal model also describes quasi-periodic motions, but with a different frequency distribution and much larger amplitudes. The differences between the two models are magnified in the second problem analyzed, where the vertical response and the dynamic tension show very different values. It is concluded that the minimal model captures the qualitative essence of the phenomenon, but is unable to describe it accurately. 


\section{Conclusions}

In this paper the dynamics of a taut string, under a traveling point-mass, either driven by an assigned horizontal force, or subjected to an assigned horizontal motion, has been studied. An exact model, previously developed by the authors, has been reconsidered and consistently simplified. Differently from almost all the literature on the subject, the nonlinear change of tension, with respect to the static pretension, has been accounted for here. For this reason, the proposed model has been called 'improved'.

Two models have been developed: (a) a massive model, governed by a partial differential equation in the transverse displacement, coupled with an ordinary differential equation in the unknown horizontal motion of the point-mass; (b) a massless model, governed by two nonlinear ordinary differential equations in the vertical and horizontal position of the point-mass. The massless model confirms the persistence of the Stokes-Smith paradox also when the point-mass has unknown motion (i.e., assigning a driving force). In these cases, the massive model point out the possible existence of 'back and forth' motions for sufficiently high values of the vertical load. The former model has been attacked by the Galerkin method, which leads to ordinary differential equations in the $N_{e}$ amplitudes of the trial function and the horizontal position $\xi$ of the point-mass. It was noticed that such result cannot be pursued by the Ritz method, if use is made of smooth trial functions, since in this way the effect of the wave resistance force is lost. Then, equations for both models, in $N_{e}+1$ or in 2 unknowns, have been numerically integrated.

Numerical simulations have been carried out, and the following conclusions can be drawn.

1. When the horizontal motion of the point-mass is assigned, the response is quite regular, except in a boundary layer close to the right-end, where all quantities suddenly increase in magnitude. Here, the effect of the change of tension, which is accounted for by the improved model, is important, since it makes stiffer the string and, therefore, limits the response value.

2. When the point-mass is moving at uniform velocity, the Stokes-Smith paradox manifests, both for massive and massless models, near the right-end support. The improved model reduces the amplitude of the gap existing between the trajectory and the support, predicted by the classical model, but it is unable to remove the paradox. A convergence study carried out for increasing number of trial functions reveals the occurrence of a peak in the horizontal displacement, close to the right end.

3. When the horizontal motion of the point-mass is unknown and the vertical force acting on the traveling mass is sufficiently small, the motion is monotonic. In this problem the influence of the distributed mass is small, so that massless model is able to capture the response at a satisfactory extent.

4. In the same case as before, but with a larger vertical force, the point-mass undergoes a nearly-periodic motion, repeatedly traveling the string in the two opposite directions ('back and forth' motions). This behavior manifests in absence of driving force too, with a small initial velocity. Here the contribution of the dynamic tension is very important, since it limits the amplitude of the nearly-periodic motions.

By summarizing, the improved model confirms the existence of 'back and forth' motions, although quantitatively different from those predicted by the minimal model. Moreover, it reduces the dynamic tension in the boundary layer. Such a model, however, is still insufficient to remove the Stokes-Smith paradox. Therefore, extensive research should be performed, taking into account all nonlinearities which have been disregarded in the studies so far carried out in literature.

\section{References}

[1] C.E. Smith, Motions of a stretched string carrying a moving mass particle, J. Appl. Mech. 31 (1)(1964) 29-37.

[2] L. Frba, Vibration of Solids and Structures under Moving Loads, vol. 1, Springer Science \& Business Media, 2013.

[3] B. Dyniewicz, C.I. Bajer, Paradox of a particle's trajectory moving on a string, Arch. Appl. Mech. 79 (3) (2009) 213-223.

[4] G.G. Stokes, Discussion of a Differential Equation Relating to the Breaking of Railway Bridges, Printed at the Pitt Press by John W. Parker, 1849.

[5] A.I. Vesnitskii, A.V. Metrikine, Transition radiation in mechanics, Phys. Usp. 39 (10)(1996) 983.

[6] A.V. Metrikine, A.R.M. Wolfert, H.A. Dieterman, Transition radiation in an elastically supported string. abrupt and smooth variations of the support stiffness, Wave Motion 27 (4) (1998) 291-305.

[7] A.V. Metrikine, Parametric instability of a moving particle on a periodically supported infinitely long string, J. Appl. Mech. 75 (1) (2008) 011006.

[8] C.I. Bajer, B. Dyniewicz, Spacetime approach to numerical analysis of a string with a moving mass, Int. J. Numer. Methods Eng. 76 (10) (2008) $1528-1543$.

[9] C.I. Bajer, B. Dyniewicz, Virtual functions of the spacetime finite element method in moving mass problems, Comput. Struct. 87 (78) (2009) $444-455$.

[10] M. Ferretti, G. Piccardo, Dynamic modeling of taut strings carrying a traveling mass, Continuum Mech. Therm. 25 (24) (2013) 469-488.

[11] L. Wang, G. Rega, Modelling and transient planar dynamics of suspended cables with moving mass, Int. J. Solids Struct. 47 (20) (2010) $2733-2744$.

[12] M. Al-Qassab, S. Nair, J. Oleary, Dynamics of an elastic cable carrying a moving mass particle, Nonlinear Dynam. 33 (1)(2003) 11-32.

[13] S.N. Gavrilov, V.A. Eremeyev, G. Piccardo, A. Luongo, A revisitation of the paradox of discontinuous trajectory for a mass particle moving on a taut string, Nonlinear Dynam. 86 (4) (2016) 2245-2260.

[14] M. Ferretti, S.N. Gavrilov, V.A. Eremeyev, A. Luongo, Nonlinear planar modeling of massive taut strings travelled by a force-driven point-mass, Nonlinear Dynam. (2019) submitted for publication.

[15] E.L. Nicolai, On pressure of vibrations, Ann. St. Petersburg Polytech. Inst. Sect. Tech. Nat. Sci. Math. 18 (1) (1912) 49-60.

[16] A.I. Vesnitski, L.E. Kaplan, G.A. Utkin, The laws of variation of energy and momentum for one-dimensional systems with moving mountings and loads, J. Appl. Math. Mech. 47 (5) (1983) 692-695.

[17] G.G. Denisov, On the wave pressure on an obstacle in the case of transversal oscillations of the string, Izv. RAN. Mek. Tverd. Tela 5 (2001) $187-192$.

[18] S.N. Gavrilov, Nonlinear investigation of the possibility to exceed the critical speed by a load on a string, Acta Mech. 154 (14)(2002) 47-60.

[19] G.G. Denisov, V.V. Novilov, M.L. Smirnova, The momentum of waves and their effect on the motion of lumped objects along one-dimensional elastic systems, J. Appl. Math. Mech. 76 (2) (2012) 225-234. 